\title{
Monotonicity of the number of positive entries in nonnegative matrix powers
}

Qimiao Xie 1* $^{*}$

\section{"Correspondence:}

qmxie@shmtu.edu.cn

${ }^{1}$ College of Ocean Science and Engineering, Shanghai Maritime

University, Shanghai, China

\section{每 Springer}

\begin{abstract}
Let $A$ be a nonnegative matrix of order $n$ and $f(A)$ denote the number of positive entries in $A$. We prove that if $f(A) \leq 3$ or $f(A) \geq n^{2}-2 n+2$, then the sequence $\left\{f\left(A^{k}\right)\right\}_{k=1}^{\infty}$ is monotonic for positive integers $k$.
\end{abstract}

MSC: $15 B 36 ; 15 B 48$

Keywords: Nonnegative matrix; Power; Monotonicity

\section{Introduction}

A matrix is nonnegative (positive) if all of its entries are nonnegative (positive) real numbers. Nonnegative matrices have many attractive properties and are important in a variety of applications [1,2]. For two nonnegative matrices $A$ and $B$ of the same size, the notation $A \geq B$ or $B \leq A$ means that $A-B$ is nonnegative.

A sign pattern is a matrix whose entries are from the set $\{+,-, 0\}$. In a talk at the 12th ILAS conference (Regina, Canada, June 26-29, 2005), Professor Xingzhi Zhan posed the following problem.

Problem ([4], p. 233) Characterize those sign patterns of square nonnegative matrices $A$ such that the sequence $\left\{f\left(A^{k}\right)\right\}_{k=1}^{\infty}$ is nondecreasing.

A nonnegative square matrix $A$ is said to be primitive if there exists a positive integer $k$ such that $A^{k}$ is positive. If we denote by $f(A)$ the number of positive entries in $A$, it seems that the sequence $\left\{f\left(A^{k}\right)\right\}_{k=1}^{\infty}$ is increasing for any primitive matrix $A$. However, Šidák [3] observed that there is a primitive matrix $A$ of order 9 satisfying $f(A)=18>f\left(A^{2}\right)=16$. This is the motivation for us to investigate the nonnegative matrix $A$ such that $\left\{f\left(A^{k}\right)\right\}_{k=1}^{\infty}$ is monotonic. It is reasonable to expect that the sequence will be monotonic when $f(A)$ is too small or too large.

Since the value of each positive entry in $A$ does not affect $f\left(A^{k}\right)$ for all positive integers $k$, it suffices to consider the $0-1$ matrix, i.e., the matrix whose entries are either 0 or 1 . Denote by $E_{i j}$ the matrix with its entry in the $i$ th row and $j$ th column being 1 and with all other entries being 0 . For simplicity we use 0 to denote the zero matrix whose size will be clear from the context.

(c) The Author(s) 2018. This article is distributed under the terms of the Creative Commons Attribution 4.0 International License (http://creativecommons.org/licenses/by/4.0/), which permits unrestricted use, distribution, and reproduction in any medium, provided you give appropriate credit to the original author(s) and the source, provide a link to the Creative Commons license, and indicate if changes were made. 


\section{Main results}

Let $A$ be a nonnegative square matrix. We will use the fact that if $A^{2} \geq A\left(A^{2} \leq A\right)$, then $A^{k+1} \geq A^{k}\left(A^{k+1} \leq A^{k}\right)$ for all positive integers $k$ and thus $\left\{f\left(A^{k}\right)\right\}_{k=1}^{\infty}$ is increasing (decreasing).

Theorem 1 Let $A$ be a 0-1 matrix of order $n$. If $f(A) \leq 2$, then the sequence $\left\{f\left(A^{k}\right)\right\}_{k=1}^{\infty}$ is decreasing.

Proof The case $f(A)=0$ is trivial.

$$
\begin{aligned}
\text { If } f(A) & =1 \text {, then } A=E_{i j}, 1 \leq i, j \leq n \text {. Thus, for } k=2,3, \ldots, \\
A^{k} & =E_{i j}^{k}= \begin{cases}E_{i i}, & i=j ; \\
0, & i \neq j,\end{cases}
\end{aligned}
$$

which implies that $\left\{f\left(A^{k}\right)\right\}_{k=1}^{\infty}$ is decreasing. Next suppose $f(A)=2$.

Since $\left\{f\left(A^{k}\right)\right\}_{k=1}^{\infty}$ is invariant under permutation similarity or transpose of $A$, it suffices to consider the following cases.

(1) $A=E_{11}+E_{22}$. Then $A^{2}=A$.

(2) $A=E_{11}+E_{12}$. Then $A^{2}=A$.

(3) $A=E_{11}+E_{23}$. Then $A^{2}=E_{11} \leq A$.

(4) $A=E_{12}+E_{13}$. Then $A^{2}=0$.

(5) $A=E_{12}+E_{21}$. Then $A^{k}=E_{11}+E_{22}$ for all even $k, A^{k}=A$ for all odd $k$.

(6) $A=E_{12}+E_{23}$. Then $A^{2}=E_{13}, A^{3}=0$.

(7) $A=E_{12}+E_{34}$. Then $A^{2}=0$.

It can be seen that in each case $\left\{f\left(A^{k}\right)\right\}_{k=1}^{\infty}$ is decreasing. This completes the proof.

Theorem 2 Let $A$ be a 0-1 matrix of order $n$. Iff $(A)=3$, then the sequence $\left\{f\left(A^{k}\right)\right\}_{k=1}^{\infty}$ is monotonic.

Proof Under permutation similarity and transpose, it suffices to consider the following cases.

(1) $A=E_{11}+E_{22}+E_{33}$. Then $A^{2}=A$.

(2) $A=E_{11}+E_{22}+E_{12}$. Then $A^{2}=A+E_{12} \geq A$.

(3) $A=E_{11}+E_{22}+E_{13}$. Then $A^{2}=A$.

(4) $A=E_{11}+E_{22}+E_{34}$. Then $A^{2}=E_{11}+E_{22} \leq A$.

(5) $A=E_{11}+E_{12}+E_{13}$. Then $A^{2}=A$.

(6) $A=E_{11}+E_{12}+E_{21}$. Then $A^{2}=A+E_{11}+E_{22} \geq A$.

(7) $A=E_{11}+E_{12}+E_{31}$. Then $A^{2}=A+E_{32} \geq A$.

(8) $A=E_{11}+E_{12}+E_{23}$. Then $A^{k}=E_{11}+E_{12}+E_{13}$ for all $k \geq 2$.

(9) $A=E_{11}+E_{12}+E_{32}$. Then $A^{2}=E_{11}+E_{12} \leq A$.

(10) $A=E_{11}+E_{12}+E_{34}$. Then $A^{2}=E_{11}+E_{12} \leq A$.

(11) $A=E_{11}+E_{23}+E_{24}$. Then $A^{2}=E_{11} \leq A$.

(12) $A=E_{11}+E_{23}+E_{32}$. Then $A^{k}=E_{11}+E_{22}+E_{33}$ for all even $k, A^{k}=A$ for all odd $k$.

(13) $A=E_{11}+E_{23}+E_{34}$. Then $A^{2}=E_{11}+E_{24}, A^{k}=E_{11}$ for all $k \geq 3$.

(14) $A=E_{11}+E_{23}+E_{45}$. Then $A^{2}=E_{11} \leq A$.

(15) $A=E_{12}+E_{13}+E_{14}$. Then $A^{2}=0$. 
(16) $A=E_{12}+E_{13}+E_{21}$. Then $A^{k}=E_{11}+E_{22}+E_{23}$ for all even $k, A^{k}=A$ for all odd $k$.

(17) $A=E_{12}+E_{13}+E_{41}$. Then $A^{2}=E_{42}+E_{43}, A^{3}=0$.

(18) $A=E_{12}+E_{13}+E_{23}$. Then $A^{2}=E_{13} \leq A$.

(19) $A=E_{12}+E_{13}+E_{24}$. Then $A^{2}=E_{14}, A^{3}=0$.

(20) $A=E_{12}+E_{13}+E_{42}$. Then $A^{2}=0$.

(21) $A=E_{12}+E_{13}+E_{45}$. Then $A^{2}=0$.

(22) $A=E_{12}+E_{21}+E_{34}$. Then $A^{k}=E_{11}+E_{22}$ for all even $k, A^{k}=E_{12}+E_{21}$ for all odd $k \geq 3$.

(23) $A=E_{12}+E_{23}+E_{31}$. Then

$$
A^{k}= \begin{cases}E_{11}+E_{22}+E_{33}, & k \equiv 0(\bmod 3) \\ A, & k \equiv 1(\bmod 3) \\ E_{13}+E_{21}+E_{32}, & k \equiv 2(\bmod 3)\end{cases}
$$

(24) $A=E_{12}+E_{23}+E_{34}$. Then $A^{2}=E_{13}+E_{24}, A^{3}=E_{14}, A^{4}=0$.

(25) $A=E_{12}+E_{23}+E_{45}$. Then $A^{2}=E_{13}, A^{3}=0$.

(26) $A=E_{12}+E_{34}+E_{56}$. Then $A^{2}=0$.

Since in each case $\left\{f\left(A^{k}\right)\right\}_{k=1}^{\infty}$ is either increasing or decreasing, this completes the proof.

Corollary 3 Let A be a 0-1 matrix of order 2. Then the sequence $\left\{f\left(A^{k}\right)\right\}_{k=1}^{\infty}$ is monotonic.

Remark When $A$ is of order $n \geq 3$ with $f(A)=4$, the following example shows that $\left\{f\left(A^{k}\right)\right\}_{k=1}^{\infty}$ may not be monotonic. Consider

$$
A=E_{12}+E_{13}+E_{21}+E_{31}
$$

Direct computation shows that

$$
A^{2}=2 E_{11}+E_{22}+E_{23}+E_{32}+E_{33}, \quad A^{3}=2 A .
$$

Thus $f(A)=4<f\left(A^{2}\right)=5>f\left(A^{3}\right)=4$.

On the one hand, Theorems 1 and 2 show that $\left\{f\left(A^{k}\right)\right\}_{k=1}^{\infty}$ is monotonic when $f(A) \leq 3$. On the other hand, $\left\{f\left(A^{k}\right)\right\}_{k=1}^{\infty}$ is expected to be also monotonic when $f(A)$ is large enough. Next we discuss the number of positive entries that $A$ has to guarantee the sequence increasing.

The permanent of a matrix $A=\left(a_{i j}\right)_{n \times n}$ is defined as

$$
\operatorname{per} A=\sum_{\sigma \in S_{n}} \prod_{i=1}^{n} a_{i, \sigma(i)},
$$

where $S_{n}$ is the set of permutations of the integers $1,2, \ldots, n$. First we have the following important fact.

Lemma 4 Let $A$ be a 0-1 matrix of order $n$. If $\operatorname{per} A>0$, then the sequence $\left\{f\left(A^{k}\right)\right\}_{k=1}^{\infty}$ is increasing. 
Proof Since $A$ is a $0-1$ matrix with per $A>0$, there exists a permutation matrix $P$ such that $A \geq P$. Now let $A=P+B$, where $B$ is also a $0-1$ matrix. Then $A^{k+1}=A \cdot A^{k}=(P+B) A^{k}=$ $P \cdot A^{k}+B \cdot A^{k} \geq P \cdot A^{k}$ for all positive integers $k$. Thus $f\left(A^{k+1}\right) \geq f\left(P \cdot A^{k}\right)=f\left(A^{k}\right)$, which implies that $\left\{f\left(A^{k}\right)\right\}_{k=1}^{\infty}$ is increasing.

Theorem 5 Let $A$ be a 0-1 matrix of order $n$. If $f(A) \geq n^{2}-2 n+2$, then the sequence $\left\{f\left(A^{k}\right)\right\}_{k=1}^{\infty}$ is increasing.

Proof First if per $A>0$, by Lemma $4,\left\{f\left(A^{k}\right)\right\}_{k=1}^{\infty}$ is increasing.

Next suppose per $A=0$. Then by the Frobenius-König theorem [4, p. 46], $A$ has an $r \times s$ zero submatrix with $r+s=n+1$. Since $f(A) \geq n^{2}-2 n+2, A$ has at most $2 n-2$ zero entries. Thus $r s \leq 2 n-2$. It can be seen that $r$ and $s$ must be one of the following solutions.

(1) $r=1, s=n$;

(2) $r=n, s=1$;

(3) $r=2, s=n-1$;

(4) $r=n-1, s=2$.

If $r=1, s=n$ or $r=n, s=1$, i.e., $A$ has a zero row or a zero column, then $A$ is permutation similar to a matrix of the form

$$
\left[\begin{array}{ll}
B & C \\
0 & 0
\end{array}\right]
$$

or its transpose, where $B$ is of order $n-1$ and $C$ is a column vector. Since $A$ has at most $2 n-2$ zero entries, $B$ has at most $n-2$ zero entries. Then there exists a permutation matrix $Q$ of order $n-1$ such that $B \geq Q$. Note that

$$
\begin{aligned}
{\left[\begin{array}{ll}
B & C \\
0 & 0
\end{array}\right]^{k+1} } & =\left[\begin{array}{ll}
B & C \\
0 & 0
\end{array}\right]\left[\begin{array}{ll}
B & C \\
0 & 0
\end{array}\right]^{k}=\left[\begin{array}{cc}
B & C \\
0 & 0
\end{array}\right]\left[\begin{array}{cc}
B^{k} & B^{k-1} C \\
0 & 0
\end{array}\right] \\
& \geq\left[\begin{array}{ll}
Q & C \\
0 & 0
\end{array}\right]\left[\begin{array}{cc}
B^{k} & B^{k-1} C \\
0 & 0
\end{array}\right]=\left[\begin{array}{cc}
Q B^{k} & Q B^{k-1} C \\
0 & 0
\end{array}\right] .
\end{aligned}
$$

Thus

$$
\begin{aligned}
f\left(A^{k+1}\right) & =f\left(\left[\begin{array}{ll}
B & C \\
0 & 0
\end{array}\right]^{k+1}\right) \geq f\left(\left[\begin{array}{cc}
Q B^{k} & Q B^{k-1} C \\
0 & 0
\end{array}\right]\right) \\
& =f\left(Q B^{k}\right)+f\left(Q B^{k-1} C\right)=f\left(B^{k}\right)+f\left(B^{k-1} C\right) \\
& =f\left(\left[\begin{array}{cc}
B^{k} & B^{k-1} C \\
0 & 0
\end{array}\right]\right)=f\left(\left[\begin{array}{cc}
B & C \\
0 & 0
\end{array}\right]^{k}\right)=f\left(A^{k}\right)
\end{aligned}
$$

for all positive integers $k$, which implies that $\left\{f\left(A^{k}\right)\right\}_{k=1}^{\infty}$ is increasing. 
If $r=2, s=n-1$ or $r=n-1, s=2$, then $A$ is permutation similar to one of the matrices $A_{1}, A_{2}, A_{1}^{T}, A_{2}^{T}$, where

$$
A_{1}=\left[\begin{array}{cccc}
0 & \cdots & 0 & 1 \\
0 & \cdots & 0 & 1 \\
1 & \cdots & 1 & 1 \\
\vdots & \ddots & \vdots & \vdots \\
1 & \cdots & 1 & 1
\end{array}\right], \quad A_{2}=\left[\begin{array}{cccc}
1 & 0 & \cdots & 0 \\
1 & 0 & \cdots & 0 \\
1 & 1 & \cdots & 1 \\
\vdots & \vdots & \ddots & \vdots \\
1 & 1 & \cdots & 1
\end{array}\right] .
$$

Direct computation shows that $A_{1}^{2} \geq A_{1}, A_{2}^{2} \geq A_{2}$. Thus $\left\{f\left(A^{k}\right)\right\}_{k=1}^{\infty}$ is increasing. This completes the proof.

Remark When $f(A)=n^{2}-2 n+1$, the following example shows that $\left\{f\left(A^{k}\right)\right\}_{k=1}^{\infty}$ may not be increasing. Consider

$$
A=\left[\begin{array}{cccc}
0 & 0 & \cdots & 0 \\
1 & 0 & \cdots & 0 \\
1 & 1 & \cdots & 1 \\
\vdots & \vdots & \ddots & \vdots \\
1 & 1 & \cdots & 1
\end{array}\right]
$$

Direct computation shows that $f(A)=n^{2}-2 n+1>f\left(A^{2}\right)=n^{2}-2 n$.

\section{Conclusion}

This paper considers the number of positive entries $f(A)$ in a nonnegative matrix $A$ and deals with the question of whether the sequence $\left\{f\left(A^{k}\right)\right\}_{k=1}^{\infty}$ is monotonic. We prove that if $f(A) \leq 3$ or $f(A) \geq n^{2}-2 n+2$, then the sequence must be monotonic. Some examples show that if $4 \leq f(A) \leq n^{2}-2 n+1$ when $n \geq 3$, then the sequence may not be monotonic.

Acknowledgements

The author would like to express her sincere thanks to referees and the editor for their enthusiastic guidance and help.

Funding

This research was supported by the National Natural Science Foundation of China (Grant No. 71503166).

Availability of data and materials

Not applicable.

\section{Competing interests}

The author declares that she has no competing interests.

Authors' contributions

The author read and approved the final manuscript.

\section{Publisher's Note}

Springer Nature remains neutral with regard to jurisdictional claims in published maps and institutional affiliations.

Received: 20 July 2018 Accepted: 1 September 2018 Published online: 21 September 2018

\section{References}

1. Bapat, R.B., Raghavan, T.E.S.: Nonnegative Matrices and Applications. Cambridge University Press, Cambridge (1997)

2. Berman, A., Plemmons, R.J.: Nonnegative Matrices in the Mathematical Sciences. SIAM, Philadelphia (1994)

3. Šidák, Z:: On the number of positive elements in powers of a non-negative matrix. Čas. Pěst. Mat. 89, 28-30 (1964)

4. Zhan, X.: Matrix Theory. Grad. Stud. Math., vol. 147. Amer. Math. Soc., Providence (2013) 